\title{
Kinematic modelling and state estimation of exploration rovers
}

\author{
Qingfeng Lou, Francisco González, József Kövecses
}

This is a post-peer-review, pre-copyedit version of an article published in IEEE Robotics and Automation Letters. The final authenticated version is available online at: http://dx.doi.org/10.1109/LRA. 2019. 2895393.

(C)2019 IEEE. Personal use of this material is permitted. Permission from IEEE must be obtained for all other uses, in any current or future media, including reprinting/republishing this material for advertising or promotional purposes, creating new collective works, for resale or redistribution to servers or lists, or reuse of any copyrighted component of this work in other works.

\begin{abstract}
State estimation is crucial for exploration rovers. It provides the pose and velocity of the rover by processing measurements from onboard sensors. Classical wheel odometry only employs encoder measurements of the two wheels in the differential drive. As a consequence, input noise can lead to large uncertainties in the estimated results. Also, the estimation models used in classical wheel odometry are nonlinear, and the linearization process that propagates the mean and covariance of the estimated state introduces additional errors in the process. This paper puts forward a novel wheel odometry approach for six-wheeled rovers. A kinematic model is formulated to relate the velocity of the wheels and the chassis, and later used to develop the corresponding estimation model. The components of the velocity of the chassis, decomposed in the chassis-fixed coordinate frame, are selected as the system state in the estimation, which results in a linear model. The motions of all wheels are fused together to provide the measurements. Wheel slip is considered random Gaussian noise in this kinematic model. The Continuous-time Kalman Filter is employed to process the model. Experimental validation with a six-wheeled rover prototype was used to confirm the validity of the proposed approach.
\end{abstract}

Keywords: Wheeled Robots, Field Robots, Kinematics, Sensor Fusion 


\section{Introduction}

State estimation is a key function to support the navigation of exploration rovers. It aims to evaluate the rover state, i.e., a set of variables that can fully describe its pose and velocity at a given instant of time, from the measurements provided by its sensors [3]. A fundamental estimation technique is based on wheel odometry, i.e., determining the state of the rover from the measurement of the motion of the wheels relative to the chassis. This technique only requires sensors that measure the rotation of the wheels, usually optical encoders, and can be used when neither global maps nor external navigation aids are available.

The state estimation problem of rovers in general has two main elements: the kinematic model and the estimation model. To connect the sensor measurements to the displacement and motion of the chassis, appropriate kinematic relations and equations need be established. This is a core element as the estimation model considers the measurement and system uncertainties based on these kinematic equations. The general approach would be to develop the kinematic model in a coordinate-system-free way; however this is not available in the literature in the most general form. Instead, the proposed solutions to formulate the kinematic model of the rover in the context of state estimation can be categorized into two main groups. The first one considers the kinematics in a very simplified way by assuming that certain points of the rover move on circular arcs, and develops the relationships for the estimation that way. A complete kinematic representation is not even developed in these approaches $[16,17]$. The second main group formulates the kinematic relations using homogeneous transformation matrices. These matrices contain the scalar components of position and velocity vectors, and rotation tensors in the global coordinate frame $[12,15]$. This makes it difficult to develop an estimation model based on the kinematic relations, since wheel encoders provide measurements that are interpreted in the local reference frame of each wheel. More complete kinematic models that consider vector representations were developed only recently in [13] and [8].

While a relatively large number of kinematic models for rover navigation exist, the estimation step has received comparatively less attention from the research community. Most models follow classical wheel odometry, e.g., $[5,11]$, in which the estimation model receives the rotation rates of the two middle wheels that function in a differential drive mode, and considers wheel slip and encoder errors as random noise. The estimation model used is nonlinear because it includes trigonometric functions to describe rover orientation. Usually, this leads to the use 
of nonlinear tools, such as the Extended Kalman Filter (EKF), to solve the estimation problem. A critical issue at this stage is the lack of redundant measurements. If no slip is assumed at the wheel/terrain interface, the measurements from the two wheels in the differential drive can only provide one unique solution for the rover state. However, pure rolling hardly ever occurs between the wheels and terrain, and the development of wheel slip results in the accumulation of significant deviations between the estimated and the true rover states. Some published studies focus on fusing the classic wheel odometry with other sensor systems to enhance the estimation performance, such as sonar [9] or IMUs [18, 6]. Others have considered the motion of all the rover wheels, merging these inputs through simplified geometric relations [2]. The Mars rover Spirit, for instance, used the average displacement of its six wheels at each time step to approximate the travelled distance [1].

This paper aims to explore what best performance can be obtained based on using purely wheel encoders and a kinematic model of the rover. It addresses two main objectives. The first is to investigate the development and performance of a systematic wheel odometry approach, particularly applied to six-wheeled rovers, in which the measurements from all wheels are fused together to provide enhanced state estimation. This element has not yet been investigated before in the context of wheeled robotic vehicles. The second main objective of this paper is to propose an approach to the kinematic representation, which allows for the development of a linear estimation model, the use of a standard Kalman filter, and through these the simplification of the estimation algorithm. The work was experimentally validated using six-wheeled rover prototypes.

\section{Methodology}

\subsection{Kinematics of a six-wheel rover}

The kinematic model developed in this section provides the relations between the velocity of the wheels and the chassis. Fig. 1 shows a six-wheeled rover that can be considered a kinematic tree composed of the ground, the chassis, and the wheels. Each wheel is connected to the chassis through a revolute joint at its steering axis, and to the ground via a contact pair, that allows both rotational and translational motion. Point $C$ denotes the geometrical centre of the chassis, and the six $W_{i}$ points, where $i=s f, p f, s m, p m, s r, p r$, are the wheel centres. In the subscripts, $s$ 


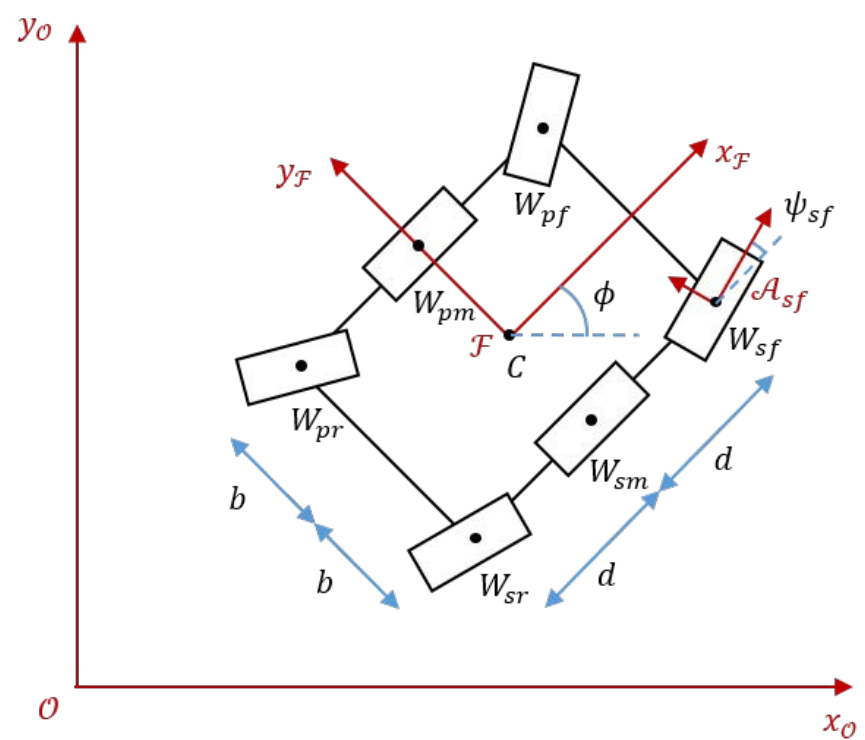

Figure 1: A planar model of a six-wheeled exploration rover

and $p$ stand for starboard and port sides, and $f, m$, and $r$ for front, middle, and rear wheels. The steering axis of each wheel goes through point $W_{i}$. Besides the ground-fixed reference frame $\mathcal{O}$ and the chassis frame $\mathcal{F}$, each wheel has a coordinate frame $\mathcal{A}_{i}$. Angle $\phi$ from axis $x_{\mathcal{F}}$ to $x_{\mathcal{O}}$ defines the orientation of the chassis. The steering angle of each wheel, $\psi_{i}$, is measured from axis $x_{\mathcal{A}_{i}}$ to $x_{\mathcal{F}}$.

The rover in Fig. 1 features six independently driven wheels. The two middle ones ( $\mathrm{pm}, \mathrm{sm}$ ) are mounted on axis $y_{\mathcal{F}}$ of the chassis and cannot be steered. They represent a differential drive; the translational and angular velocity of the chassis can be controlled by their different rotation rates. The four corner wheels ( $p f, s f, p r$, and $s r$ ) are steered following the Ackermann criterion, such that their axes intersect at a common point located on the axis of the two middle wheels.

The absolute position vector of a wheel centre $W_{i}, \vec{p}_{W_{i}}$, can be expressed as

$$
\vec{p}_{W_{i}}=\vec{p}_{C}+\vec{p}_{W_{i} / C}
$$

where $\vec{p}_{C}$ is the absolute position vector of $C$, and $\vec{p}_{W_{i} / C}$ is the position difference between $W_{i}$ and $C$. Differentiating Eq. (1) with respect to time, gives the velocity of $W_{i}$ as

$$
\vec{v}_{W_{i}}=\vec{v}_{C}+\vec{\omega}_{c} \times \vec{p}_{W_{i} / C}
$$

where $\vec{v}_{C}$ is the velocity of $C$ and $\vec{\omega}_{c}$ is the angular velocity of the chassis. The algebraic repre- 
sentation of Eq. (2) can be established decomposing each vector in $\mathcal{F}$ as

$$
\mathbf{v}_{W_{i}}^{\mathcal{F}}=\mathbf{v}_{C}^{\mathcal{F}}+\omega_{c}^{\mathcal{F}} \times \mathbf{p}_{W_{i} / C}^{\mathcal{F}}
$$

where terms $\mathbf{v}_{W_{i}}^{\mathcal{F}}, \mathbf{v}_{C}^{\mathcal{F}}, \omega^{\mathcal{F}}$ and $\mathbf{p}_{W_{i} / C}^{\mathcal{F}}$ are $3 \times 1$ column matrices and contain the $x, y$, and $z$ coordinates of each vector in frame $\mathcal{F}$. Eq. (3) can be rearranged as

$$
\mathbf{v}_{W_{i}}^{\mathcal{F}}=\left[\begin{array}{ll}
\mathbf{I} & -\tilde{\mathbf{P}}_{W_{i} / C}^{\mathcal{F}}
\end{array}\right]\left[\begin{array}{c}
\mathbf{v}_{C}^{\mathcal{F}} \\
\omega_{c}^{\mathcal{F}}
\end{array}\right]
$$

where $\mathbf{I}$ is the $3 \times 3$ identity matrix and $\tilde{\mathbf{P}}_{W_{i} / C}^{\mathcal{F}}$ is the skew-symmetric cross product matrix associated with $\mathbf{p}_{W_{i} / C}^{\mathcal{F}}$. The components of the velocity of $W_{i}$ decomposed in frame $\mathcal{A}_{i}$ can be obtained through the velocity transformation

$$
\mathbf{v}_{W_{i}}^{\mathcal{A}_{i}}=\mathbf{R}_{\mathcal{F}}^{\mathcal{A}_{i}} \mathbf{v}_{W_{i}}^{\mathcal{F}}=\mathbf{R}_{\mathcal{F}}^{\mathcal{A}_{i}}\left[\begin{array}{ll}
\mathbf{I} & -\tilde{\mathbf{P}}_{W_{i} / C}^{\mathcal{F}}
\end{array}\right]\left[\begin{array}{c}
\mathbf{v}_{C}^{\mathcal{F}} \\
\omega_{c}^{\mathcal{F}}
\end{array}\right]
$$

where $\mathbf{R}_{\mathcal{F}}^{\mathcal{A}_{i}}$ is the rotation matrix from $\mathcal{F}$ to $\mathcal{A}_{i}$.

The rover is a three-dimensional mechanical system, and the above equations are for the general case. However, if the rover moves on terrain that can be considered flat then main estimation problem can be addressed with a planar model as show in Fig. 1, where the reference plane is presented by the $x$ and $y$ axes of the different coordinate frames. It is assumed that the steering axes are perpendicular to this reference plane during motion. The motion of the chassis can then be described with a set of three velocity components, $\mathbf{u}_{C}=\left[\begin{array}{lll}v_{C_{x}} & v_{C_{y}} & \omega_{c}\end{array}\right]^{\mathrm{T}}$, where $v_{C_{x}}$ and $v_{C_{y}}$ are the components of the velocity of point $C$, and $\omega_{c}$ is the component of the angular velocity of the chassis perpendicular to the plane of motion considered. Based on Eq. (5) for this planar model, the velocity of a wheel centre can be expressed in terms of $\mathbf{u}_{C}^{\mathcal{F}}$ as

$$
\mathbf{u}_{W_{i}}^{\mathcal{A}_{i}}=\left[\begin{array}{c}
v_{W_{i_{x}}} \\
v_{W_{i_{y}}}
\end{array}\right]^{\mathcal{A}_{i}}=\left[\begin{array}{ll}
\mathbf{S}_{\mathcal{F}}^{\mathcal{A}_{i}} & \mathbf{t}_{W_{i} / C}
\end{array}\right] \mathbf{u}_{C}^{\mathcal{F}}
$$

where $\mathbf{S}_{\mathcal{F}}^{\mathcal{A} i}$ is the $2 \times 2$ rotation matrix that corresponds to the relative rotation between frames $\mathcal{F}$ and $\mathcal{A}_{i}$ and $\mathbf{t}_{W_{i} / C}$ is the $2 \times 1$ velocity difference term that results from the $-\mathbf{R}_{\mathcal{F}}^{\mathcal{A}_{i}} \tilde{\mathbf{P}}_{W_{i} / C}^{\mathcal{F}}$ product 
in Eq. (5). Eq. (6) can be written more compactly as

$$
\mathbf{u}_{W_{i}}^{\mathcal{A}_{i}}=\mathbf{J}_{i} \mathbf{u}_{C}^{\mathcal{F}}
$$

where $\mathbf{J}_{i}=\left[\begin{array}{cc}\mathbf{S}_{\mathcal{F}}^{\mathcal{A}_{i}} & \mathbf{t}_{W_{i} / C}\end{array}\right]$. Grouping the velocities of all wheels in a single $12 \times 1$ array $\mathbf{u}_{W}^{\mathcal{A}}$ we can write

$$
\mathbf{u}_{W}^{\mathcal{A}}=\mathbf{J} \mathbf{u}_{C}^{\mathcal{F}}
$$

where

$$
\left.\mathbf{u}_{W}^{\mathcal{A}}=\left[\begin{array}{lll}
\left(\begin{array}{cc}
\mathbf{u}_{W_{s f}}^{\mathcal{A}_{s f}}
\end{array}\right)^{\mathrm{T}} & \cdots & \left(\mathbf{u}_{W_{p r}}^{\mathcal{A}_{p r}}\right.
\end{array}\right)^{\mathrm{T}}\right]^{\mathrm{T}}
$$

and $\mathbf{J}$ is a $12 \times 3$ matrix composed of the corresponding six $\mathbf{J}_{i}$ terms. Eq. (8) represents the relation between the absolute velocity of the centre of each wheel, decomposed in its frame $\mathcal{A}_{i}$, and the velocity of point $C$ of the chassis decomposed in frame $\mathcal{F}$. The wheel/ground no-slip constraints are not imposed in Eq. (8); the wheels can develop slip in both longitudinal and lateral directions.

If no-slip constraints are imposed at the wheel/ground interface, then $v_{C y}=0$ and Eq. (7) would become

$$
\left[\begin{array}{c}
r_{W} \dot{\theta}_{i} \\
0
\end{array}\right]=\mathbf{J}_{i}\left[\begin{array}{c}
v_{C_{x}} \\
0 \\
\omega_{c}
\end{array}\right]^{\mathcal{F}}
$$

where $r_{W}$ is the wheel radius and $\dot{\theta}_{i}$ is the rotation rate of wheel $i$ about its axis. Using Eq. (10) the rotation rates of the two middle wheels can be related to the velocity of the chassis as

$$
\left[\begin{array}{c}
r_{W} \dot{\theta}_{s m} \\
r_{W} \dot{\theta}_{p m}
\end{array}\right]=\left[\begin{array}{cc}
1 & b \\
1 & -b
\end{array}\right]\left[\begin{array}{l}
v_{C_{x}} \\
\omega_{c}
\end{array}\right]^{\mathcal{F}}
$$

which is the kinematic model for the differential drive used in classical odometry. Eq. (11) indicates that if no-slip constraints are imposed, the velocity of the chassis is uniquely determined for a given value of $\dot{\theta}_{s m}$ and $\dot{\theta}_{p m}$. Similar equations can be written for each of the corner wheels; 
for $i=s f$, Eq. (7) takes the form

$$
\left[\begin{array}{c}
r_{W} \dot{\theta}_{i} \\
0
\end{array}\right]=\left[\begin{array}{cc}
\cos \psi_{i} & b \cos \psi_{i}+d \sin \psi_{i} \\
-\sin \psi_{i} & -b \sin \psi_{i}+d \cos \psi_{i}
\end{array}\right]\left[\begin{array}{c}
v_{C_{x}} \\
\omega_{c}
\end{array}\right]^{\mathcal{F}}
$$

Eq. (12) or its counterparts for $p f, s r$, and $p r$ can be used to find the velocity of the chassis from the rotation rate of each corner wheel. Therefore, the velocity of the chassis can be obtained in five different ways from wheel measurements using Eqs. (11) and (12).

\subsection{Estimation based on wheel odometry}

The odometry-based estimation model is intended to fuse the encoder measurements from all the wheels, filtering out random noises, and providing the estimated rover state. The main technique used for estimation is the Kalman Filter (KF) concept, which was designed for discretetime linear systems. Extensions and generalizations have also been developed, such as the Continuous-time Kalman Filter (CKF) that works with continuous-time linear systems, the Extended Kalman Filter (EKF) and the Unscented Kalman Filter (UKF) that are able to deal with nonlinear systems. Detailed descriptions of these techniques can be found in [4]. This section develops the estimation model in a linear continuous-time form starting with the kinematic model in Eq. (8), using the CKF concept.

The general form of a zero-input linear continuous-time system [14] can be written as

$$
\begin{array}{ll}
\dot{\mathbf{x}}=\mathbf{A x}+\mathbf{w}_{s} ; & \text { where } \mathbf{w}_{s} \sim\left(\mathbf{0}, \mathbf{Q}_{c}\right) \\
\mathbf{y}=\mathbf{C x}+\mathbf{v}_{s} ; & \text { where } \mathbf{v}_{s} \sim\left(\mathbf{0}, \mathbf{R}_{c}\right)
\end{array}
$$

Eq. (13a) is the system equation, where $\mathbf{x}$ is the $n \times 1$ system state vector and $\mathbf{A}$ is the $n \times n$ state matrix. Eq. (13b) is the measurement equation, where $\mathbf{y}$ is the observed $m \times 1$ measurement vector, and $\mathbf{C}$ is an $m \times n$ matrix giving the ideal (noiseless) connection between the measurement and the system state. The system noise is represented by an $n \times 1$ vector $\mathbf{w}_{s}$, assumed to be uncorrelated, zero-mean, white noise with Gaussian distribution. It has a known $n \times n$ covariance matrix $\mathbf{Q}_{c}$. A large variance of a component of $\mathbf{w}_{s}$ means that the confidence in the corresponding system equation is small and vice versa. The measurement noise is represented by $m \times 1$ vector $\mathbf{v}_{s}$, also assumed to be Gaussian noise with a known $m \times m$ covariance matrix 
$\mathbf{R}_{c}$. A large variance of a component of $\mathbf{v}_{s}$ means small confidence in the measurement accuracy.

The first step in the development of the rover estimation model is the selection of an appropriate system state $\mathbf{x}$. Rover state and system state in this context are two different terms. Rover state refers to the pose and velocity of the chassis decomposed in the absolute coordinate frame $\mathcal{O}$, whereas system state refers to the variables used in the estimation model in Eqs. (13).

In classical odometry, the system state consists in the position and orientation of the chassis in $\mathcal{O}$, and the longitudinal and angular velocity of the chassis in $\mathcal{F}[5,11]$, such that

$$
\mathbf{x}^{\mathrm{T}}=\left[\begin{array}{lllll}
p_{C_{x}}^{\mathcal{O}} & p_{C_{y}}^{\mathcal{O}} & \phi & v_{C_{x}}^{\mathcal{F}} & \omega_{c}^{\mathcal{F}}
\end{array}\right]
$$

The nonlinear estimation model of classical odometry is expanded as

$$
\left[\begin{array}{c}
p_{C_{x}}^{\mathcal{O}} \\
p_{C_{y}}^{\mathcal{O}} \\
\phi \\
v_{C_{x}}^{\mathcal{F}} \\
\omega_{c}^{\mathcal{F}}
\end{array}\right]_{k+1}=\left[\begin{array}{c}
p_{C_{x}}^{\mathcal{O}}+\Delta t v_{C_{x}}^{\mathcal{F}} \cos \left(\phi+\frac{\Delta t \omega_{c}^{\mathcal{F}}}{2}\right) \\
p_{C_{y}}^{\mathcal{O}}+\Delta t v_{C_{x}}^{\mathcal{F}} \sin \left(\phi+\frac{\Delta t \omega_{c}^{\mathcal{F}}}{2}\right) \\
\phi+\Delta t \omega_{c}^{\mathcal{F}} \\
v_{C_{x}}^{\mathcal{F}} \\
\omega_{c}^{\mathcal{F}}
\end{array}\right]_{k}\left[\begin{array}{c}
n_{x} \\
n_{y} \\
n_{\phi} \\
n_{v} \\
n_{\omega}
\end{array}\right]_{k}
$$

$$
\left[\begin{array}{c}
r_{W} \dot{\theta}_{s m} \\
r_{W} \dot{\theta}_{p m}
\end{array}\right]=\left[\begin{array}{ccccc}
0 & 0 & 0 & 1 & b \\
0 & 0 & 0 & 1 & -b
\end{array}\right]\left[\begin{array}{c}
p_{C_{x}}^{\mathcal{O}} \\
p_{C_{y}}^{\mathcal{O}} \\
\phi \\
v_{C_{x}}^{\mathcal{F}} \\
\omega_{c}^{\mathcal{F}}
\end{array}\right]_{k}
$$

where $\Delta t$ is the data sampling period. This selection of the system state is straightforward since the rover pose can be obtained directly after solving the estimation problem. However, such a selection inevitably leads to a nonlinear estimation model because of the presence of trigonometric functions to describe the rover orientation $\phi$. Although the nonlinear problem can be solved with an EKF, the linearization process introduces additional computational cost and estimation errors. 
Here we propose a new selection for the system state in the estimation problem, with which a linear estimation model can be developed. The proposed system state includes the velocity components of the chassis expressed in its body-fixed coordinate frame $\mathcal{F}$ and can be written as

$$
\mathbf{x}^{\mathrm{T}}=\left[\begin{array}{lll}
v_{C_{x}} & v_{C_{y}} & \omega_{c}
\end{array}\right]^{\mathcal{F}}=\left(\mathbf{u}_{C}^{\mathcal{F}}\right)^{\mathrm{T}}
$$

Two main differences exist between these two possible selections. First, the absolute position of the chassis is not included in the system state in Eq. (17); the rover kinematic model (8) is defined at the velocity level only and the corresponding estimation model can also be established at the velocity level. The observed measurement in this case consists of the wheel rotation rates obtained from the encoders. Wheel slip is considered as random noise. Accordingly, the estimation will not be affected by the removal of the position information of the chassis. Second, both the longitudinal and lateral velocity components of the chassis in the chassis-fixed frame $\mathcal{F}$ are included in (17).

The system state proposed in (17) cannot be integrated directly over time. After solving the estimation problem, extra post-processing is needed to transfer the estimated system state to the required rover state. However, this can be done in a simple and efficient way.

The new selection of system state brings about several advantages. Transforming the velocity of the chassis from $\mathcal{F}$ into $\mathcal{O}$ at each iteration of the estimation process is not required, which removes the need to determine the chassis orientation. Therefore, the nonlinear estimation model required by classical odometry is replaced by a linear model. Moreover, the lateral velocity of the chassis can be estimated. These improvements contribute to making the estimation more simple, robust, and accurate.

Because the velocity components used for the chassis do not depend on the position and orientation, $\mathbf{A}=\mathbf{0}$ in Eq. (13a), which reduces to

$$
\dot{\mathbf{x}}=\mathbf{w}_{s}=\left[\begin{array}{lll}
a_{x} & a_{y} & \alpha
\end{array}\right]^{\mathrm{T}}
$$

where $a_{x}, a_{y}$, and $\alpha$ can be interpreted as randomly varying rates. The expression of the measurement equation (13b) can be obtained from Eq. (8). The absolute velocity of the centre of 
each wheel $i$ is given by

$$
\mathbf{u}_{W_{i}}^{\mathcal{A}_{i}}=\left[\begin{array}{c}
v_{W_{i x}} \\
v_{W_{i y}}
\end{array}\right]^{\mathcal{A}_{i}}=\left[\begin{array}{c}
r_{W} \dot{\theta}_{i}-\dot{\zeta} \\
\dot{\eta}
\end{array}\right]=\left[\begin{array}{c}
r_{W} \dot{\theta}_{i} \\
0
\end{array}\right]+\left[\begin{array}{c}
-\dot{\zeta}_{i} \\
\dot{\eta}_{i}
\end{array}\right]
$$

where $\dot{\zeta}$ and $\dot{\eta}$ represent the wheel slip components in the longitudinal and lateral directions. From Eqs. (7) and (19),

$$
\boldsymbol{\rho}_{i}=\mathbf{J}_{i} \mathbf{x}+\boldsymbol{\xi}_{i}
$$

where $\boldsymbol{\rho}_{i}=\left[\begin{array}{ll}r_{W} \dot{\theta}_{i} & 0\end{array}\right]^{\mathrm{T}}$ and $\boldsymbol{\xi}_{i}=\left[\begin{array}{cc}-\dot{\zeta}_{i} & \dot{\eta}_{i}\end{array}\right]^{\mathrm{T}}$. Grouping the components for the six wheels of the rover results in

$$
\rho=\mathbf{J}_{\mathbf{X}}+\boldsymbol{\xi}
$$

which provides the expression of the measurement equation (13b), where $\mathbf{y}=\boldsymbol{\rho}, \mathbf{C}=\mathbf{J}$, and $\mathbf{v}_{s}=\boldsymbol{\xi}$. Rates $\dot{\theta}_{i}$ in term $\boldsymbol{\rho}$ are directly obtained from encoder readings. The wheel slip components $\dot{\zeta}_{i}$ and $\dot{\eta}_{i}$ are treated as random noise that affects the measurements.

Eqs. (18) and (21) form a linear continuous-time estimation model. It can be transformed into a discrete-time form based on the encoder sampling frequency, and solved with the Discretetime Kalman Filter (DKF). In practice, the proposed odometry can be fused with other sensor systems such as IMU, visual odometry, or motor torque. In this case, non-uniform sampling frequency or analytical analysis might be required. To keep the generality of the method, the Continuous-time Kalman Filter [7] was used in this paper.

The initial condition at time $t=0$ can be established as

$$
\begin{aligned}
& \hat{\mathbf{x}}(0)=E[\mathbf{x}(0)] \\
& \mathbf{P}(0)=E\left[(\mathbf{x}(0)-\hat{\mathbf{x}}(0))(\mathbf{x}(0)-\hat{\mathbf{x}}(0))^{\mathrm{T}}\right]
\end{aligned}
$$

where $E[\cdot]$ stands for the expected value of a term. $E[\mathbf{x}(0)]$ is the expected value of the true system state $\mathbf{x}$ at time $t=0$. Before any measurement is available, it is reasonable to set the estimated state as this value. $\mathbf{P}$ is an $n \times n$ matrix that denotes the covariance of the estimation error. The recursive differential equations used to solve the estimated state in the CKF are given 
as [7]

$$
\begin{aligned}
& \mathbf{K}=\mathbf{P} \mathbf{C}^{\mathrm{T}} \mathbf{R}_{c}^{-1} \\
& \dot{\hat{\mathbf{x}}}=\mathbf{A} \hat{\mathbf{x}}+\mathbf{K}(\mathbf{y}-\mathbf{C} \hat{\mathbf{x}}) \\
& \dot{\mathbf{P}}=-\mathbf{P} \mathbf{C}^{\mathrm{T}} \mathbf{R}_{c}^{-1} \mathbf{C P}+\mathbf{A} \mathbf{P}+\mathbf{P} \mathbf{C}^{\mathrm{T}}+\mathbf{Q}_{c}
\end{aligned}
$$

where term $\mathbf{K}$ is the Kalman gain, which depends only on system parameters $\mathbf{A}, \mathbf{C}, \mathbf{R}_{\mathbf{c}}$, and $\mathbf{Q}_{\mathbf{c}}$, and is not affected by the measurement $\mathbf{y}$. Eq. (23b) indicates that $\mathbf{K}$ defines the rate of change of the estimated state $\hat{\mathbf{x}}$ with respect to $\mathbf{y}$. A larger gain makes the update of $\hat{\mathbf{x}}$ more dependent on the most recent measurements, while a lower gain causes the update to depend more on the system equation.

Eq. (23c) is a Continuous-time Algebraic Riccati Equation (CARE), from which the covariance of the estimation error $\mathbf{P}$ can be solved for. However, solving Eq. (23c) through direct numerical integration can be computationally expensive. Here we use the transition matrix approach [10] as an alternative, which provides a more efficient way to obtain $\mathbf{P}$.

The use of different variables to define the system state and the rover state requires the introduction of an additional step at each iteration of the CKF algorithm. The rover state at time step $k$ must be obtained from its value in the previous step, $k-1$, and the estimated system state at step $k$. First, the chassis orientation in reference frame $\mathcal{O}$ is calculated by integrating the angular velocity of the chassis.

$$
\phi_{k}=\phi_{k-1}+\int_{k-1}^{k} \omega_{c} \mathrm{~d} t
$$

Then, the velocity of the chassis in $\mathcal{O}$ is calculated using the corresponding rotation matrix $\mathbf{R}_{\mathcal{F}}^{\mathcal{O}}\left(\phi_{k}\right)$,

$$
\mathbf{u}_{C_{k}}^{\mathcal{O}}=\mathbf{R}_{\mathcal{F}}^{\mathcal{O}} \mathbf{u}_{C_{k}}^{\mathcal{F}}
$$

The absolute position of the chassis in $\mathcal{O}$ can be found as

$$
\mathbf{p}_{C_{k}}^{\mathcal{O}}=\mathbf{p}_{C_{k-1}}^{\mathcal{O}}+\int_{k-1}^{k} \mathbf{u}_{C_{k}}^{\mathcal{O}} \mathrm{d} t
$$



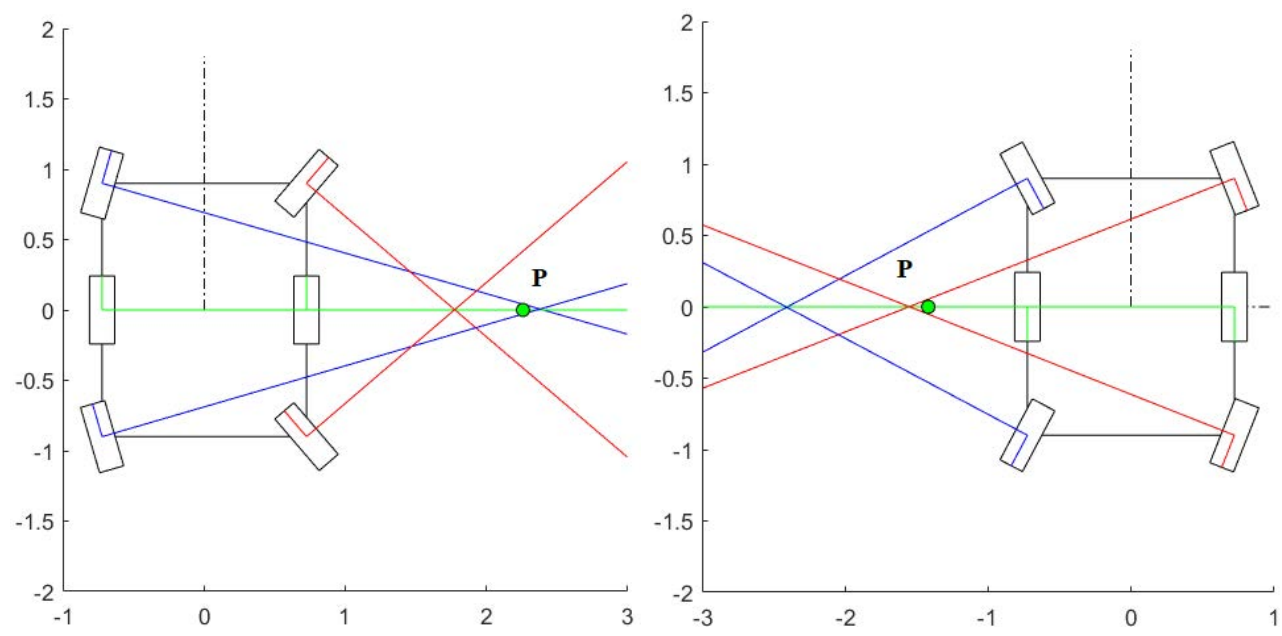

Figure 2: Centres of rotation of the chassis predicted using different pairs of wheels

\section{Experimental validation and results}

The methodology described in Section 2 was validated experimentally using two different sixwheeled rover prototypes. Results from the experiments illustrate the advantage of fusing measurements from different wheels to estimate the rover state. As discussed in Section 2.1 the rover state can be determined in five different ways from the readings of the encoders if no-slip at the wheel/terrain interface is assumed, using Eq. (11) or Eq. (12). Using these, it is possible to find the instant centre of rotation that the chassis would have if wheel slip were zero. Fig. 2 shows two typical rover configurations in left and right side turns. The instant centre of rotation of the chassis that corresponds to the motion of the middle wheels is located at point P. Two additional locations for the centre of rotation can be found as the intersection of the axes of the port or starboard wheels, and the axis of the differential drive wheels. Ideally, these three points should always be coincident. In practice they are not, because the steering angles and rotation rates of the wheels differ from the ideal steering condition due to errors introduced by terrain reaction forces and response delays in control, among other reasons. A different chassis motion will be predicted depending on which wheels are selected for the analysis. On the other hand, as shown in Section 2.2, a unique rover state can be determined fusing the readings of all its encoders using the estimation model defined in Eqs. (18) and (21), where the no-slip constraints are not assumed.

It must be noted that the proposed odometry cannot be used to correct deviations derived from wheel slip that is compatible with the rover kinematic relations. An example of this is all the wheels of the rover developing the same longitudinal slip during motion along a straight 


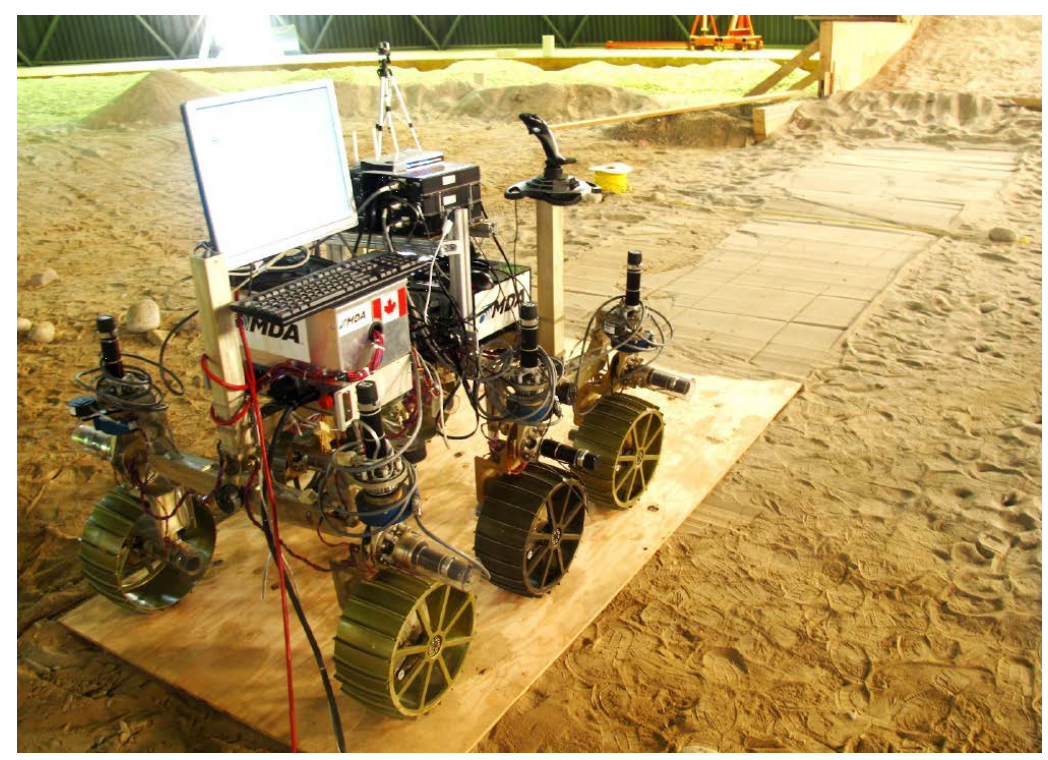

Figure 3: The RCP rover used in experimental validation

line. The proposed odometry that fuses all wheels will also contain the same bias. On the other hand, the proposed odometry is able to remove the bias caused by individual wheel slip that is not compatible with the kinematic relations. Such slip is a common source of errors when the vehicle is turning.

\subsection{First set of experiments}

A first set of experiments was carried out with the Rover Chassis Prototype (RCP), shown in Fig. 3, developed and owned by MacDonald, Dettwiler and Associates (MDA). The RCP is composed of a main body attached to three bogies (port, starboard, and rear) via passive revolute joints. Each bogie has two independently steered and driven wheels. The locomotion configuration in Fig. 1 can be used to describe the motion of the RCP on flat terrain.

Experiments for testing the developed odometry were carried out in the Mars Dome of the University of Toronto Institute for Aerospace Studies (UTIAS). This is a covered testing field specially designed for rover evaluation. As shown in Fig. 4, a $6.2 \mathrm{~m} \times 6.2 \mathrm{~m}$ square path was marked on flat soft terrain. The RCP was manually guided to follow the selected path clockwise. Encoder measurements of the wheel rotation rates $\dot{\theta}_{i}$ and steering angles $\psi_{i}$ were collected with a sampling period of $\Delta t=0.1 \mathrm{~s}$ via the RCP data acquisition system. The manoeuvre was completed in about $500 \mathrm{~s}$. 


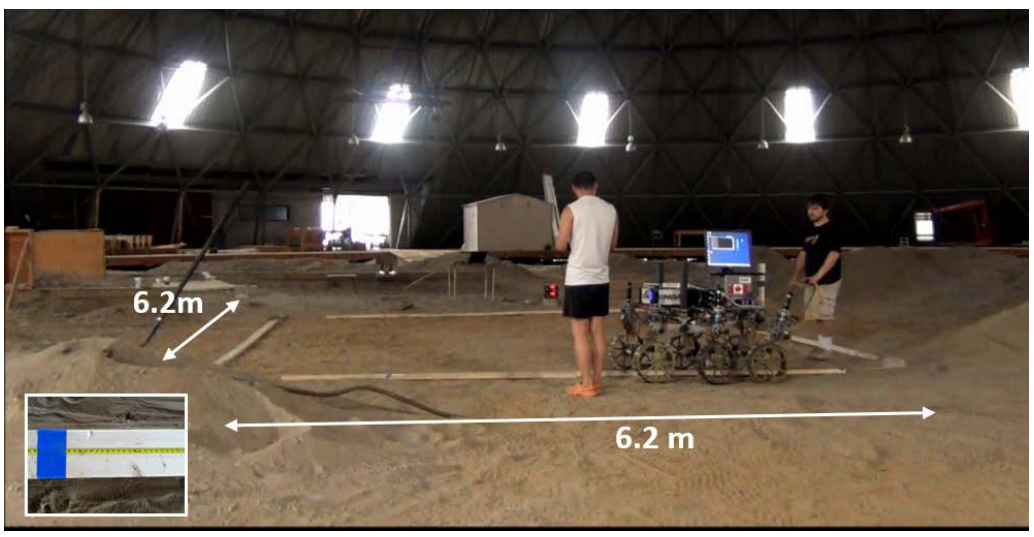

Figure 4: Experimental setup

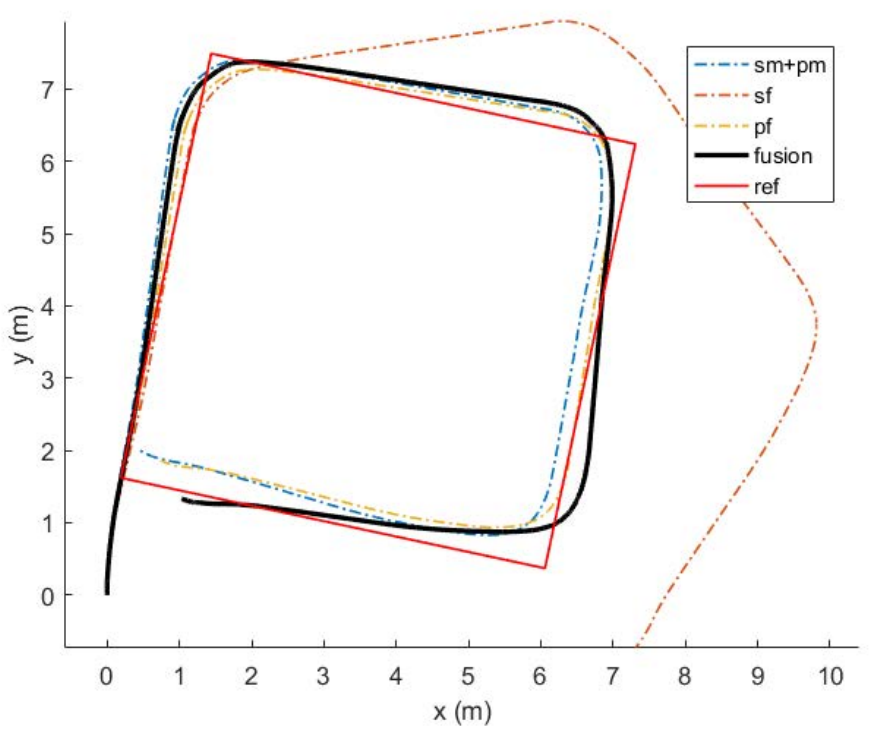

Figure 5: Trajectory of point $C$ of the RCP chassis

Fig. 5 shows the absolute position of point $C$ of the chassis obtained with different odometry approaches; the square path represents the goal trajectory. Results from the encoder readings of the wheels on the differential drive and Eq. (11) are labelled ' $s m+p m$ '. Counterparts of Eq. (12) were applied to the readings from the port and starboard front wheels to determine the values labelled ' $p f$ ' and ' $s f$ '. The results obtained with the proposed algorithm, which fuses information from all the wheels following the KF approach introduced in Section 2.2, are denoted by 'fusion'. This estimation method effectively fused information from all wheels and removed the deviation due to the measurements from the $s f$ wheel. 


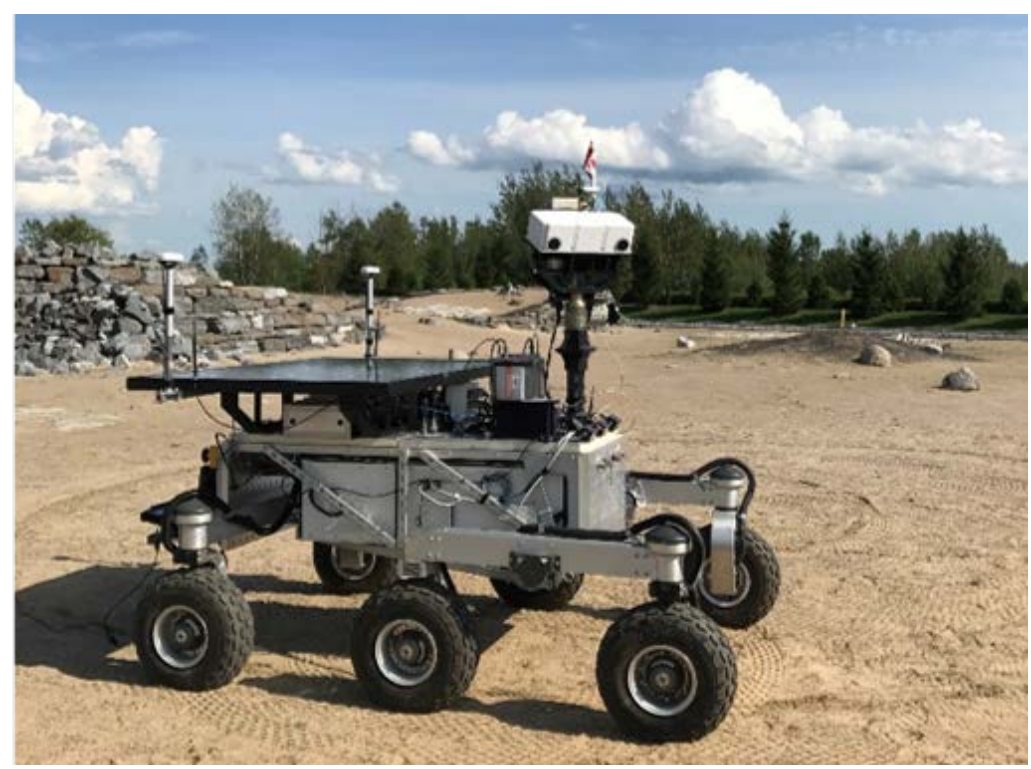

Figure 6: Mars Exploration Science Rover (MESR) of CSA

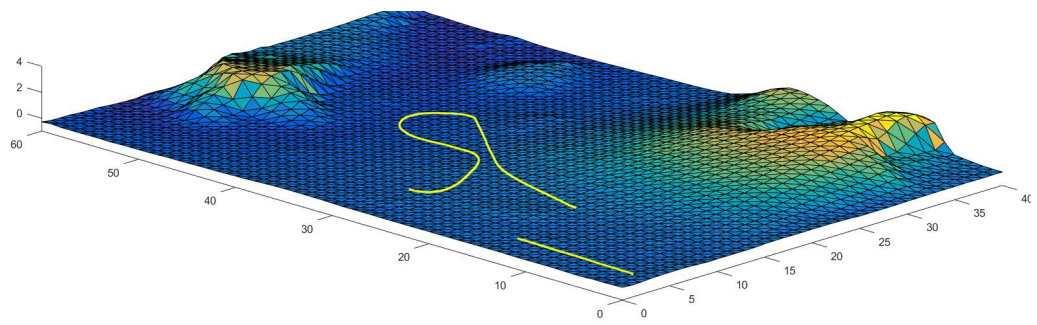

Figure 7: Mars Emulation Terrain (MET) at CSA

\subsection{Second set of experiments}

A second set of experiments was conducted using the Canadian Space Agency's (CSA) Mars Exploration Science Rover (MESR), shown in Fig. 6, which was also developed by MDA. The locomotion configuration of the MESR is the same one as RCP, and can be described with Fig. 1 as well.

The navigation experiments were carried out at the Mars Emulation Terrain (MET) of the Canadian Space Agency (CSA), shown in Fig. 7. This sandy terrain contains features that are representative of the operations environment on Mars.

The MESR was manually guided to travel on a relatively flat sector of the MET. The position and orientation of the chassis were recorded by a Differential Global Positioning System (DGPS) sampled at $1 \mathrm{~Hz}$. Although the DGPS has a certain amount of measurement error, it converges at the position level and does not grow as the travelled distance increases. The DGPS readings are therefore considered as ground truth in this experiment set. Twelve encoders, sampled at 


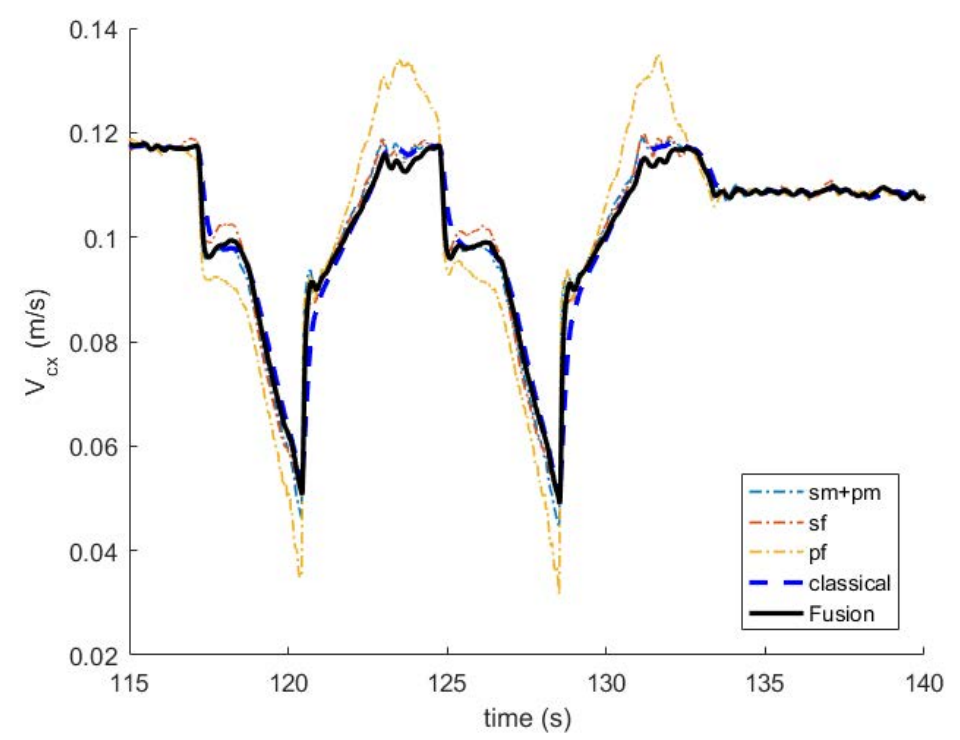

Figure 8: Chassis longitudinal velocity during manoeuvre 2

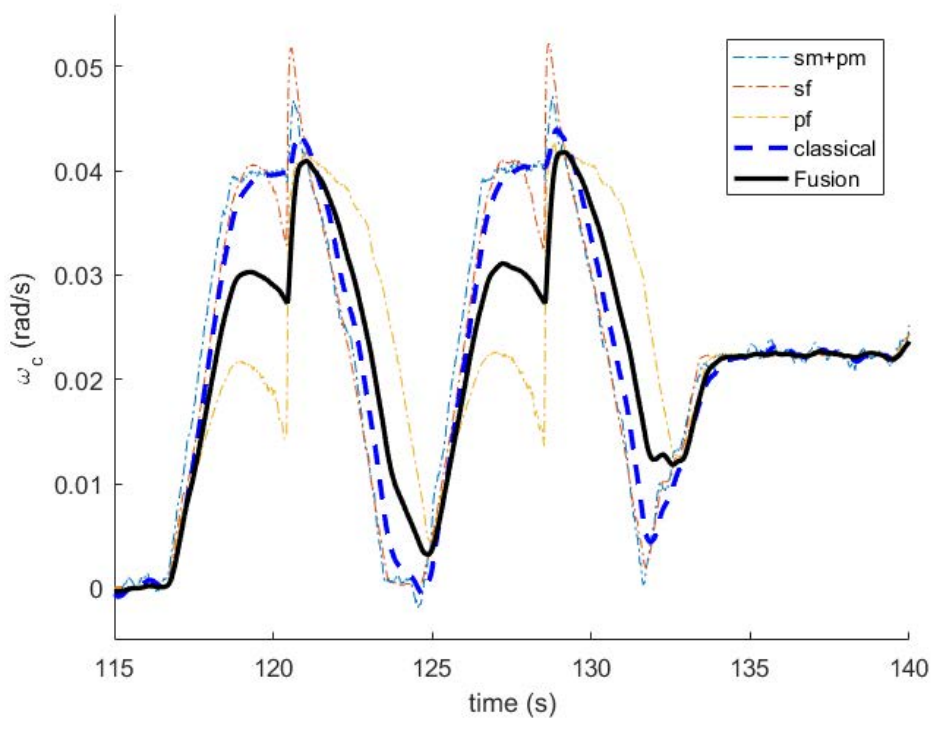

Figure 9: Chassis angular velocity during manoeuvre 2

$50 \mathrm{~Hz}$, provided the rotation rates and steering angles of the wheels. Results from two paths, highlighted in Fig. 7, are shown in this Section: a straight line to check the slip ratio of the wheels (manoeuvre 1), and a random curve to test the proposed odometry (manoeuvre 2).

In the first manoeuvre, the rover was driven along a straight line for $12 \mathrm{~m}$. The forward velocity of the MESR was determined using data recorded by the DGPS. The rover velocity was also determined using readings from the wheel encoders, assuming rolling at the wheel-terrain interface. The comparison between the two values indicated a stable slip ratio of $4.35 \%$. 


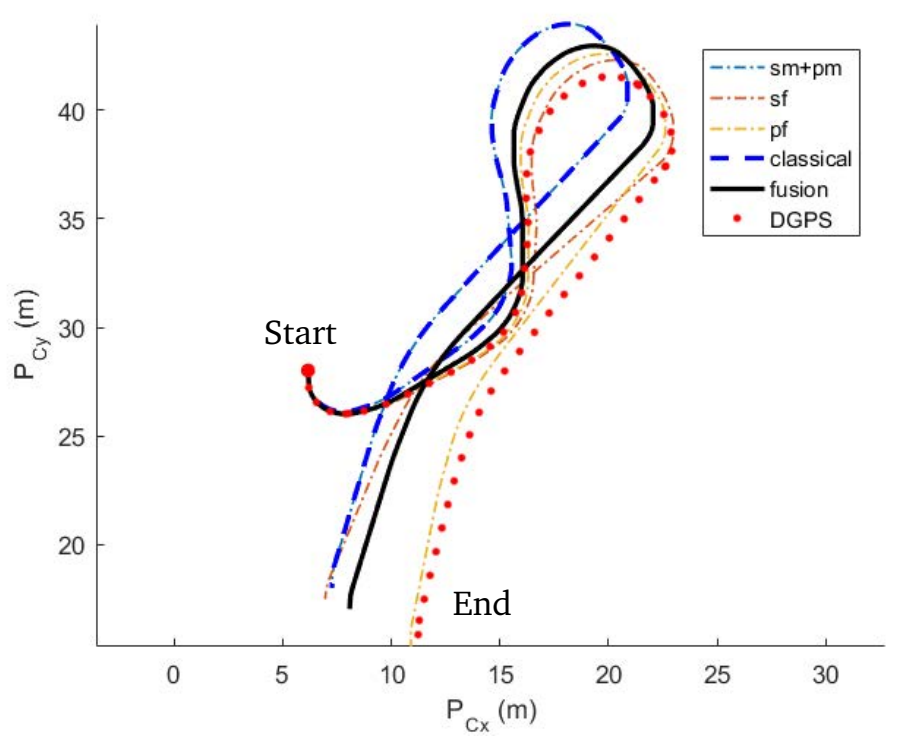

Figure 10: Chassis position during manoeuvre 2

Figs. 8 and 9 show the $v_{C_{x}}$ and $\omega_{c}$ components of the velocity of the chassis $\mathbf{u}_{C}^{\mathcal{F}}$ between $t=115 \mathrm{~s}$ and $t=140 \mathrm{~s}$ in manoeuvre 2 , obtained using the odometry approaches introduced in Section 3.1. Results from classical odometry obtained from the application of an EKF to the encoder readings of the two middle wheels are also included in the comparison and labelled as 'classical'.

At $t=115 \mathrm{~s}$ the rover started a right-side turn. Figs. 8 and 9 show that the velocity of the chassis obtained from ' $s m+p m$ ', ' $s f$ ', and ' $p f$ ' readings have significant differences, which indicates the existence of conflicting measurements at different wheels. Classical odometry associated with EKF only used readings from the two middle wheels. Although it smoothed the differences that stemmed from the ' $s m+p m$ ' readings, its prediction still followed the ' $s m+p m$ ' measurements within a small boundary. The 'fusion' approach, on the other hand, did not require the linearization of the estimation model and provided the estimation results determined by the probability distribution of the slip of all wheels.

The differences between the evaluated odometry approaches are shown in Fig. 10, which contains the trajectory described by the rover in the second manoeuvre, relative to the global coordinate frame $\mathcal{O}$. The rover travelled around $60 \mathrm{~m}$ in $600 \mathrm{~s}$. The 'classical' and 'sm $+\mathrm{pm}$ ' results overlapped almost completely, as expected, since the motion of the two middle wheels is the only input in both cases. On the other hand, the 'fusion' results incorporate the results from all the wheels. Fig. 11 shows the chassis orientation determined using the different methods, 


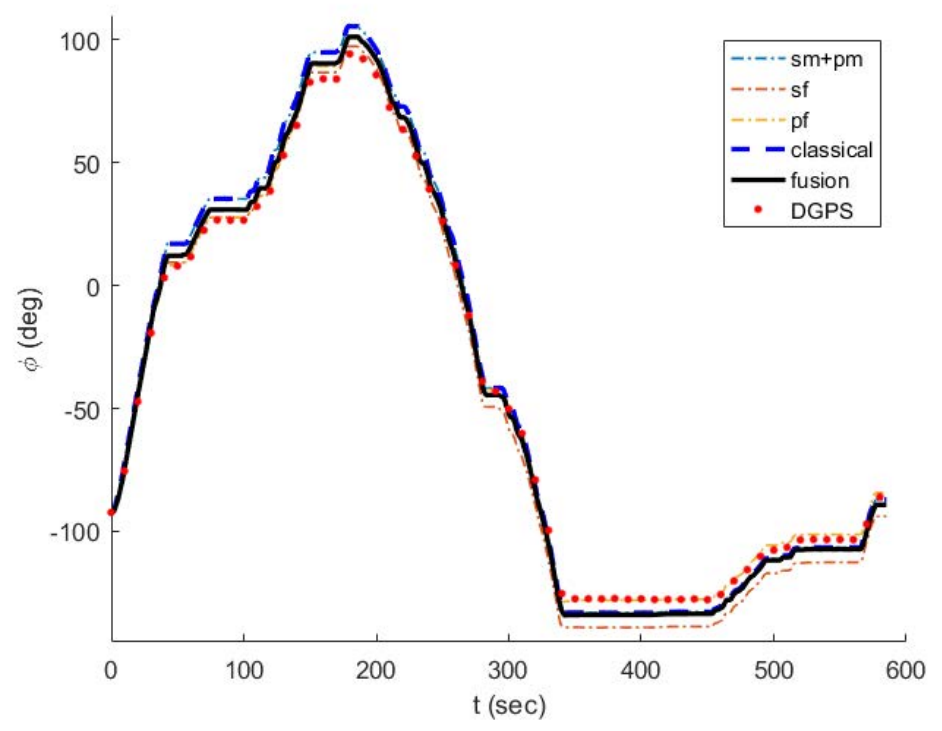

Figure 11: Chassis orientation during manoeuvre 2

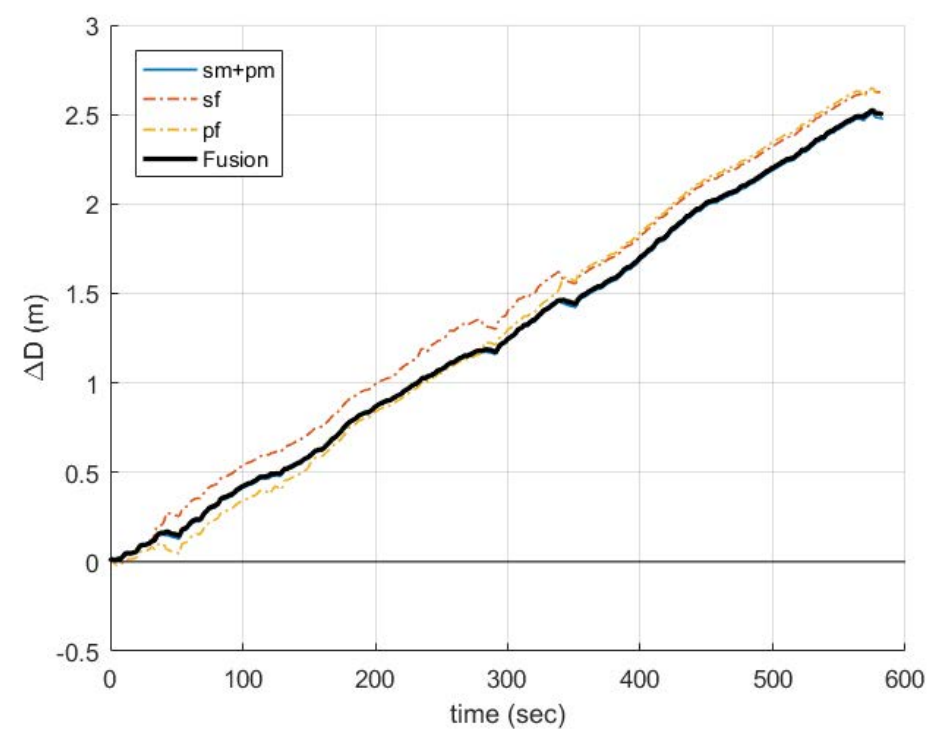

Figure 12: Distance error $\Delta D$

which generally followed the DGPS readings within a difference below $20^{\circ}$.

Figs. 12 and 13 show two indicators of the estimation performance. Errors $\Delta D$ and $\Delta \phi$ measure the difference between the travelled distance and the rover orientation estimated with each method and the reference value provided by the DGPS. Line ' $s m+p m$ ' stands for both 'classical' and 'sm $+p m$ ' methods in both figures. In Fig. 13, $\Delta \phi$ was reset to zero at $t=180 \mathrm{~s}$, when the MESR transitioned from counter-clockwise to clockwise rotation.

The distance error $\Delta D$ was similar for all the methods. It is proportional to longitudinal 


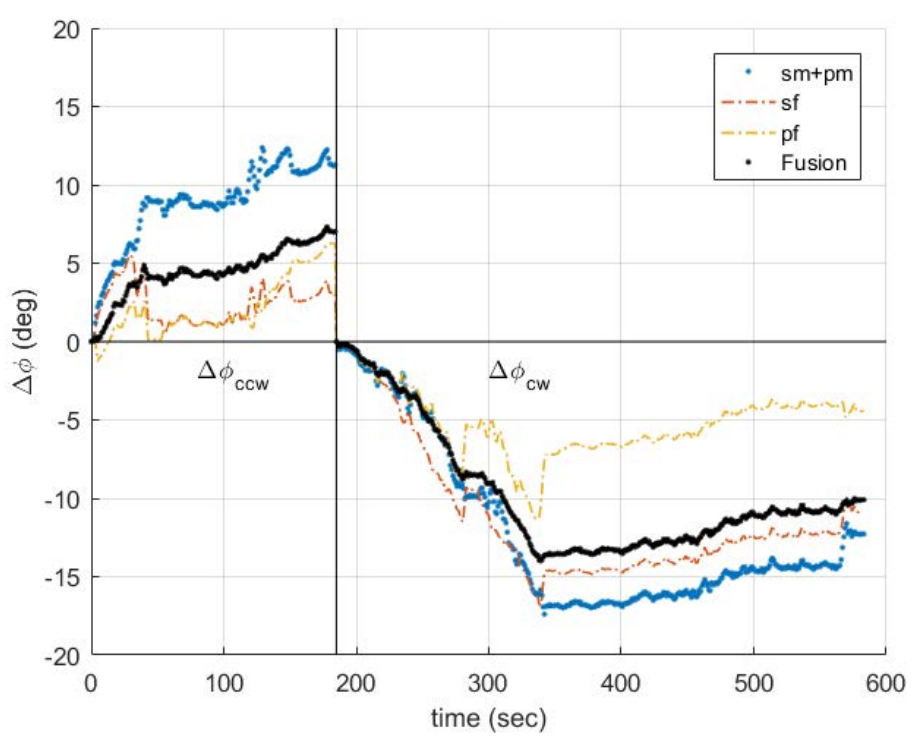

Figure 13: Orientation error $\Delta \phi$

slip, which affected every wheel in a similar way. Purely kinematics-based odometry cannot be used to correct this error. The orientation error $\Delta \phi$, on the other hand, varied significantly depending on the correction approach used. Classical odometry resulted in $\Delta \phi_{c c w}=13^{\circ}$ and $\Delta \phi_{c w}=17^{\circ}$. The 'fusion' method reduced these values to $6^{\circ}$ and $13^{\circ}$, respectively. This resulted in an improved tracking of the ground truth trajectory derived from the DGPS reading, as also shown in Fig. 10.

The method proposed in this paper has two main elements, namely, a kinematic modelling approach combined with a regular Kalman filter, and the fusing of the redundant measurements from multiple encoders. These could also be applied to the classical odometry approach separately.

Applying our kinematic modelling approach to the two middle wheels also leads to a linear estimation model with non-redundant inputs; a regular Kalman filter can then be used for this. We tested this and the estimation results obtained were found to be very close to what the classical odometry delivers. However, for our approach linearization is not required, and the implementation and computations are more efficient.

The classical odometry has a different modelling approach as highlighted by Eqs. (15) and (16). It would require the further development of that approach to fuse the readings from all the wheel encoders and carry out the estimation using the EKF; such a model has not yet been reported in the literature. One possible way for that development can be to replace the mea- 
surement equation (16) with the kinematic relations defined in Eq. (8), and neglect the lateral velocities of the wheels and the chassis. This would lead to a nonlinear estimation model associated with the EKF approach that would fuse inputs from all wheel encoders. However, even if that approach is developed, it would include more approximations and assumptions than the method we proposed in this paper.

\section{Conclusions}

This paper introduces a novel method for state estimation of rovers that uses wheel encoders as the only measurements. While classical wheel odometry relies only on the two wheels in the differential drive, the proposed method fuses information from all the wheels in the rover. The method consists of two elements. First, a rover kinematic model is developed to represent the relation between the wheel velocity components in the wheel-fixed coordinate frames and the velocity of the chassis decomposed in the chassis-fixed frame. Wheel/ground constraints are not imposed in this model so that the possibility of developing longitudinal and lateral slip is included in the relations. Second, a new estimation model is formulated and solved using the CKF algorithm. The integration of the velocity of the chassis is separated from the estimation, and its components in the chassis-fixed coordinate frame $\mathcal{F}$ are selected as system state. By doing so, the position and velocity of the chassis no longer need to be decomposed in the ground-fixed coordinate frame during the estimation process, which makes it possible to develop a linear estimation model instead of the nonlinear one used in classical wheel odometry. Experiments with two rover prototypes were conducted to test the proposed method. Results indicate that readings from multiple encoders can be successfully fused together. The errors introduced by individual wheel measurements are effectively eliminated, and the uncertainties caused by slip are reduced. Although the proposed wheel odometry method is based on a typical six-wheeled rover configuration, it is also applicable for other wheeled rovers and vehicles on either hard or soft terrain. The proposed technique can also be fused with other sensor measurements such as from IMUs and visual sensors. 


\section{Acknowledgements}

This work was supported by the Natural Sciences and Engineering Research Council of Canada, and MacDonald, Dettwiler and Associates Space Missions. F. González was funded by the Ministry of Economy of Spain through the Ramón y Cajal programme, contract no. RYC-2016-20222. The experiments were supported by the Canadian Space Agency.

The authors would like to thank Andrew Gibson, Pierre-Alexandre Desrochers, David Gingras, David Shaffer and their team at the CSA and Drs. Joseph Bakambu and Bahareh Ghotbi of MDA for their assistance with the experiments using the MESR rover, and Prof. Tim Barfoot and his team at UTIAS for their help during the experiments with the RCP rover.

\section{References}

[1] Ali, K.S., Vanelli, C.A., Biesiadecki, J.J., Maimone, M.W., Cheng, Y., San Martin, A.M., Alexander, J.W.: Attitude and position estimation on the Mars exploration rovers. In: 2005 IEEE Int. Conf. on Systems, Man and Cybernetics, vol. 1, pp. 20-27 (2005)

[2] Baumgartner, E.T., Aghazarian, H., Trebi-Ollennu, A.: Rover localization results for the FIDO rover. In: Intelligent Systems and Advanced Manufacturing, pp. 34-44 (2001)

[3] Borenstein, J., Feng, L., Everett, H.R.: Navigating Mobile Robots: Systems and Techniques. AK Peters, Wellesley, MA, USA (1996)

[4] Brown, R.G., Hwang, P.Y.C.: Introduction to Random Signals and Applied Kalman Filtering. John Wiley \& Sons, Hoboken, NJ, USA (1997)

[5] Chong, K.S., Kleeman, L.: Accurate odometry and error modelling for a mobile robot. In: IEEE Int. Conf. on Robotics and Automation (ICRA), vol. 4, pp. 2783-2788 (1997)

[6] Iqbal, J., Rehman-Saad, M., Malik, A., Mahmood-Tahir, A.: State estimation technique for a planetary robotic rover. Revista Facultad de Ingeniería Universidad de Antioquia (73), 58-68 (2014)

[7] Kalman, R.E., Bucy, R.S.: New results in linear filtering and prediction theory. Journal of Basic Engineering 83(1), 95-108 (1961) 
[8] Kelly, A., Seegmiller, N.: A vector algebra formulation of mobile robot velocity kinematics. In: Field and Service Robotics, vol. 92, pp. 613-627 (2014)

[9] Kleeman, L.: Advanced sonar and odometry error modeling for simultaneous localisation and map building. In: IEEE/RSJ Int. Conf. on Intelligent Robots and Systems (IROS), vol. 1, pp. 699-704 (2003)

[10] Lancaster, P., Rodman, L.: Algebraic Riccati Equations. Clarendon Press, Oxford, UK (1995)

[11] Larsen, T.D., Hansen, K.L., Andersen, N.A., Ravn, O.: Design of Kalman filters for mobile robots; evaluation of the kinematic and odometric approach. In: 1999 IEEE Int. Conf. on Control Applications, vol. 2, pp. 1021-1026 (1999)

[12] Muir, P.F., Neuman, C.P.: Kinematic modeling of wheeled mobile robots. Journal of Robotic Systems 4(2), 281-340 (1987)

[13] Pol, S.: Odometry for a planetary exploration rover. Master's thesis, KTH (2007)

[14] Simon, D.: Optimal state estimation: Kalman, H infinity, and nonlinear approaches. John Wiley \& Sons, Hoboken, NJ, USA (2006)

[15] Tarokh, M., McDermott, G.J.: Kinematics modeling and analyses of articulated rovers. IEEE Transactions on Robotics 21(4), 539-553 (2005)

[16] Tonouchi, Y., Tsubouchi, T., Arimoto, S.: Fusion of dead-reckoned positions with a workspace model for a mobile robot by Bayesian inference. In: IEEE/RSJ/GI Int. Conf. on Intelligent Robots and Systems (IROS), vol. 2, pp. 1347-1354 (1994)

[17] Wang, C.M.: Location estimation and uncertainty analysis for mobile robots. In: Autonomous Robot Vehicles, pp. 90-95. Springer (1990)

[18] Zunaidi, I., Kato, N., Nomura, Y., Matsui, H.: Positioning system for 4-wheel mobile robot: encoder, gyro and accelerometer data fusion with error model method. CMU Journal 5(1), $1-14(2006)$ 